\title{
Les cimetières musulmans en Espagne : des lieux de l'altérité
}

The Muslim Cemeteries in Spain: Spaces of Otherness

Los cementerios musulmanes en España: otros espacios de la alteridad

Jordi Moreras et Sol Tarrés

\section{(2) OpenEdition}

Journals

\section{Édition électronique}

URL : https://journals.openedition.org/remi/5993

DOI : 10.4000/remi.5993

ISSN : $1777-5418$

\section{Éditeur}

Université de Poitiers

Édition imprimée

Date de publication : 1 septembre 2012

Pagination : 13-26

ISBN : 979-10-90426-05-4

ISSN : 0765-0752

\section{Référence électronique}

Jordi Moreras et Sol Tarrés, «Les cimetières musulmans en Espagne : des lieux de l'altérité », Revue européenne des migrations internationales [En ligne], vol. 28 - n³ | 2012, mis en ligne le 01 septembre 2015, consulté le 14 avril 2022. URL : http://journals.openedition.org/remi/5993 ; DOI : https://doi.org/ 10.4000/remi.5993 


\section{Les cimetières musulmans en Espagne : des lieux de l'altérité}

\section{Jordi Moreras ${ }^{1}$ et Sol Tarrés ${ }^{2}$}

" Jamais les morts n'ont été, pour les urbanistes, aussi encombrants"

(Thomas, $1975: 355)$

\section{Introduction}

Dans les années 1970, la planification de nouveaux cimetières dans les grandes villes européennes répond au trop-plein des anciens cimetières urbains, à l'aménagement de nouveaux terrains où les corps sont disposés différemment (les niches superposées se généralisent) et à de nouveaux services funéraires. Aujourd'hui, l'intérêt pour la planification urbaine se déplace vers l'agencement des espaces intérieurs des cimetières, afin de tenir compte de la diversité des dimensions culturelles, religieuses et émotionnelles de la mort dans notre société.

Par définition, les cimetières sont des endroits pluriels où la diversité (culturelle, sociale, religieuse) tente de surmonter l'uniformité qui accompagne la mort (Baudry, 1999). Cette affirmation peut paraître évidente, mais elle met l'accent sur l'idée que la diversité est présente dans les cimetières au moment de l'enterrement des immigrés, ces morts " différents". Chaque société sait comment enterrer ses morts, mais pas toujours comment enterrer ses " autres morts ". L'histoire du cimetière reflète la façon dont chaque société a dû faire face à l'autre mort : celle de ceux qui avaient des croyances différentes de celles de la majorité, ceux dont les comportements brisaient la morale sociale, ceux qui ont décidé de mettre fin à leur vie, ou ceux qui, tout simplement, étaient des étrangers.

Aujourd'hui, la société espagnole se demande à nouveau où mettre ses " autres morts ". Elle le fait dans le cadre d'une approche distincte et inclusive, preuve de sa pluralisation croissante, alors que reconnaître la diversité religieuse dans le domaine funéraire et réserver des terrains dans les cimetières publics pour les différentes communautés religieuses est devenu une " bonne pratique " administrative.

Dans le cas des communautés musulmanes, ces exigences sont confrontées à un aspect inattendu qui joue un rôle symbolique de premier ordre : l'existence de cimetières pour les musulmans, liée à la présence historique de cette population comme c'est le

1 Departament d'Antropologia, Filosofia i Treball Social, Facultat de Lletres de Tarragona, Universitat Rovira i Virgili, Av. Catalunya 35, 43002 Tarragona, Espagne ; jordi.moreras@urv.cat

2 Departamento de Historia I, Facultad de Humanidades. Pabellón 12, planta Baja, Campus de «El Carmen», Avenida de las Fuerzas Armadas, s/n, 21071 Huelva, Espagne ; sol.tarres@dhis1.uhu.es 
cas à Ceuta ${ }^{3}$, ou à la création, par les insurgés, pendant la guerre civile espagnole (19361939), d'espaces pour enterrer les troupes marocaines recrutées. Aujourd'hui, des défunts musulmans sont inhumés dans ces cimetières et l'appropriation de ces lieux fait resurgir une mémoire historique presque oubliée : la participation des troupes coloniales dans la guerre civile espagnole.

Aborder l'étude de ces "lieux " réservés à l'inhumation musulmane dans les cimetières publics ne peut se faire du seul point de vue de la gestion de la diversité religieuse liée à l'immigration. II convient également de prendre en compte d'autres facteurs historiques et symboliques inscrits dans les mémoires : celles de la société espagnole, des familles et des pratiques communautaires des Marocains en Espagne, mises à l'épreuve par la mort de l'un des leurs. Mourir en migration est un élément inattendu, une variable imprévue qui est, malgré tout, révélatrice de l'altération de l'ordre implicite du binôme émigration-immigration, comme le soulignait Abdelmalek Sayad (2000:9) : " la mort de l'immigré est à l'image et à la mesure de ce que fut sa vie. Elle est une mort qui dérange tout le monde, car elle est une mort inclassable [...], parce qu'elle rappelle à chacun et elle rappelle pour tout le monde ce qu'est la double condition de I'homme immigré ici et émigré de là ".

La mort se transforme alors, pour celui qui décède, en un lien généalogique au sens ambivalent. Être inhumé dans cette société d'accueil, ou décider de renvoyer le corps vers le lieu d'origine, entraîne des interprétations très différentes vis-à-vis de la condition ambiguë de "l'intégration " de la part des membres de la société d'accueil : la première option est vue comme le point culminant d'un long processus entamé du vivant du défunt, renforcé par un ultime témoignage de loyauté consistant à être enterré dans la terre d'accueil ; la seconde est perçue comme la preuve définitive d'une intégration manquée. Choisir d'être inhumé ici ou là peut être interprété comme une trahison des accords que suppose la double condition d'émigré/immigré. Mais la mort signifie aussi la fin des exigences de loyauté imposées par les sociétés d'accueil et d'origine.

Dans le cadre de cette réflexion sur l'interprétation de la mort en contexte d'immigration, cet article se propose de rendre compte de huit expériences attachées à ces espaces d'inhumation musulmane en Espagne et de décrire les enjeux liés à la gestion de ces "lieux $"^{4}$.

\section{Les enjeux juridiques et historiques de la séparation entre les tombes}

La loi espagnole, dans son article 16 de la Constitution espagnole de 1978, reconnaît le droit pour tous les citoyens à des funérailles selon leurs convictions religieuses, philosophiques ou culturelles. II est complété par la loi organique de liberté religieuse (1980) qui, explicitement (article 2.1b), garantit le droit de chacun à " recevoir une sépulture digne, sans discrimination pour des motifs religieux ". Ce principe avait déjà été reconnu par la loi 49/1978 du 3 novembre relative à l'inhumation dans les cimetières locaux (art. 1). Le but de cette loi était de mettre fin à la discrimination subie par les citoyens noncatholiques dans un État confessionnel catholique avec des cimetières gérés par l'Église (Rodriguez Blanco, 2010).

3 Enclave espagnole située sur la côte marocaine, face à Gibraltar.

4 Les données présentées dans ce texte ont été recueillies dans le cadre d'un projet de recherche sur la mort, les migrations et la diversité, impliquant les Universités Rovira i Virgili, Huelva et Barcelone. Voir aussi Tarrés, Solé, Jàvega et Moreras, 2012. 
L'esprit de cette loi de 1980 est le résultat de I'histoire religieuse espagnole des XIXe et XXe siècles (Jiménez Lozano, 1981). On pourrait la décrire comme une succession de réformes, motivées par les changements politiques, destinées à maintenir le caractère catholique des cimetières ou alors à les désacraliser. II s'agissait de bâtir ou de démolir des murs séparant les défunts ayant connu une " bonne mort " (en accord avec les principes de la doctrine catholique) de ceux l'ayant transgressée, tous ceux qui étaient " indignes de recevoir la sépulture ecclésiastique " (suicidés, athées déclarés et noncatholiques). C'est le roi réformiste Carlos III (1716-1788) qui décréta le caractère public des cimetières, en attribuant la charge de la gestion et des services funéraires aux municipalités, au détriment des paroisses catholiques. Au milieu du XIXe siècle, la création des cimetières civils s'est généralisée en Espagne pour enterrer tous les morts, catholiques ou pas.

La responsabilité progressive prise par les administrations civiles dans la gestion de I'inhumation en dehors de l'Église catholique, a été validée par la loi du 30 janvier 1932, adoptée lors de la Seconde République, laquelle stipule que les "cimetières civils seraient communs à tous les citoyens, sans différences fondées sur des raisons religieuses ". Les rites funéraires et les symboles religieux devaient se limiter à chaque tombe ; les murs qui séparaient les parties confessionnelles des parties civiles devaient être démolis. Mais en 1938, en pleine guerre civile, le gouvernement provisoire du général Franco a abrogé cette loi, revenant sur la question des murs de séparation entre la partie du cimetière dite catholique et celle qui regroupait les tombes dites civiles. Cette séparation allait alors s'inscrire de façon visible dans l'espace des cimetières locaux, les tombes civiles étant disposées dans les lieux les plus périphériques et les plus discrets. Avec la réinstauration de la démocratie en Espagne, cette séparation a finalement été supprimée par la loi 49/1978 qui stipule que les cimetières locaux cessent d'être religieux ${ }^{5}$.

Les Accords de coopération signés en 1992 entre l'Espagne et des représentants juifs et musulmans (lois 25/1992 et 26/1992, respectivement) ont développé le cadre juridique de la liberté religieuse, incorporant une référence directe à la question de l'inhumation en fonction des traditions religieuses (art. 2.5). Cet article reconnaît le droit de " disposer de parcelles réservées aux sépultures islamiques dans les cimetières municipaux ", et d'adopter des " mesures appropriées pour l'application des règles traditionnelles islamiques ", à condition qu'elles soient " conformes aux dispositions de la législation municipale et de la santé publique ". Il a été complété par d'autres règlements liés aux services funéraires, qui ne prennent pas toujours en considération les spécificités de la tradition islamique. Ces règlements ont cependant suscité de nombreuses discussions. Tout d'abord, celle concernant l'obligation d'utiliser un cercueil pour l'inhumation, conformément à la réglementation sanitaire qui s'applique en Espagne, à l'exception de l'Andalousie où, en 2001, a été adoptée une loi permettant la sépulture religieuse directement dans le sol, sans cercueil. Depuis, cette disposition est évoquée par les représentants musulmans désireux de faire appliquer le même critère dans d'autres régions d'Espagne. Ensuite, celle concernant la séparation entre les tombes qui, bien que reconnue explicitement dans l'Accord de 1992, n'est pas précisée dans le détail. En I'absence de critères,

5 Dans de nombreux cimetières espagnols, on trouve encore des témoignages de commémoration de la guerre civile, par la présence de monuments et de tombeaux érigés par les vainqueurs. Dans le contexte de l'adoption par le Parlement espagnol de la loi sur la mémoire historique en 2007, certains cimetières ont voulu créer des lieux de mémoire, afin de rappeler la perte irremplaçable des vies humaines dans le conflit. 
les juristes estiment que cette séparation n'a pas à être très marquée ${ }^{6}$. Des auteurs comme Motilla (2004) interrogent sur la nature de ces séparations, liées au principe de sécularisation des cimetières, tandis que d'autres les justifient au motif du principe de la liberté religieuse (Rodríguez Blanco, 2010) ${ }^{7}$. Ce débat fait écho au passé récent des cimetières en Espagne. En effet, l'enterrement séparé des juifs et des musulmans ne peut pas être présenté comme un fait " nouveau " alors qu'il relève d'une pratique ancienne. Cependant, celle-ci prend une signification très différente aujourd'hui puisqu'on propose de séparer pour reconnaître, alors qu'auparavant, il s'agissait simplement d'écarter ceux qui avaient choisi une " autre " mort en transgressant la norme majoritaire.

\section{Entre la mémoire historique et la gestion actuelle du plura- lisme}

Il convient de rappeler que l'Espagne a une histoire de sept siècles de présence musulmane sur son sol, ce qui la singularise au regard d'autres pays d'Europe. Pour la société espagnole, le passé interfère avec son présent (Viguera, 1995), tandis que pour les communautés musulmanes, il apparaît comme une référence identitaire plus ou moins active, en particulier chez les musulmans convertis. L'histoire prend une dimension symbolique que la société espagnole a mobilisée de façon différente au cours des dernières décennies : en tant que patrimoine historique et modèle de coexistence interculturelle ou comme argument néo-conservateur contre cette mystification identitaire. Parfois, ce rappel du passé sert aussi à se situer par rapport à la situation de l'islam en Espagne. Bien qu'il soit pratiquement impossible d'établir une relation entre ces deux processus historiques séparés par treize siècles, des voix tentent cependant d'établir un parallèle entre l'entrée des troupes arabo-berbères dirigées par Tariq Ibn Zyad dans la Péninsule lbérique en l'an 711 et l'installation de migrants depuis les années $1960^{8}$.

Malgré la reconnaissance de l'Accord de coopération concernant les inhumations entre l'Espagne et la Commission islamique d'Espagne, la réservation de parcelles dans les cimetières municipaux espagnols n'est pas encore une pratique généralisée. Nous avons répertorié vingt-huit cimetières où procéder à une inhumation musulmane. Les carrés sont de dimensions modestes et paraissent, a priori, insuffisants pour répondre aux besoins de près d'un million et demi de personnes.

La gestion actuelle de la mort musulmane en Espagne trouve un écho dans le passé récent. À Ceuta et Melilla ${ }^{9}$, rares territoires européens avec une présence musulmane de longue date, la question des sépultures islamiques n'est pas toujours bien résolue. Le premier cimetière musulman à Ceuta date du XVIIle siècle et I'actuel, placé à côté du sanctuaire de Sidi Embarek, fait l'objet d'une réforme depuis 2010, avec pour projet de l'agrandir sur un terrain appartenant à l'armée espagnole. À Melilla, jusqu'en 1992, les défunts musulmans étaient inhumés sur le sol marocain. Le cimetière actuel, de Sidi Guariach, est situé à côté de la frontière, séparé par un simple mur du cimetière marocain.

\footnotetext{
6 Le guide Recommandations pour la gestion de la diversité religieuse dans les cimetières, édité par le gouvernement régional de Catalogne en 2009, suggère de ne pas utiliser n'importe quel élément de séparation qui crée " une ségrégation sévère ", sans pourtant proposer aucun exemple. 7 La législation des cimetières prévoit que les ordres monastiques ou les associations religieuses peuvent avoir leurs propres parcelles, mais sans qu'il y ait une séparation formelle et à condition de maintenir une continuité avec le reste du cimetière.

8 Pour une vision globale et actualisée de la présence musulmane en Espagne, voir Moreras (2011). 9 Comme Ceuta (note 3), Melilla est aussi une enclave espagnole au Maroc, située sur la côte marocaine, très proche de l'Algérie.
} 
Le deuxième appel à l'histoire concerne les cimetières militaires créés pendant la guerre civile espagnole ${ }^{10}$ et tirés de l'oubli, dans les années 1970, par des musulmans espagnols avec l'intention de les réutiliser. C'est notamment le cas de Grenade et de Séville. Le cimetière de Griñon (Madrid), également été utilisé pendant la guerre civile, a été donné au consulat marocain à Madrid, par l'armée espagnole, à la fin des années 1970. D'autres cimetières, comme Cordoue et Saragosse ont été récemment rouverts (en 1992 et 2002, respectivement ; le premier dans une parcelle différente de la parcelle d'origine), tandis que ceux de Barcia (Asturies), La Coruña et Cáceres, fermés après la guerre civile, ont fait l'objet d'initiatives de restauration.

Tableau 1 : Les cimetières musulmans en Espagne

\begin{tabular}{|l|l|}
\hline \multicolumn{1}{|c|}{ Date } & \multicolumn{1}{c|}{ Ville } \\
\hline XVIIle siècle & Ceuta \\
\hline $1936-1939 *$ & $\begin{array}{l}\text { Grenade (2003) } \\
\text { Séville (1987) } \\
\text { Griñon-Madrid (1978) } \\
\text { Saragosse (2002) } \\
\text { Burgos (2009) } \\
\text { León (2010) }\end{array}$ \\
\hline 1992 & $\begin{array}{l}\text { Melilla } \\
\text { Cordoue }\end{array}$ \\
\hline 1996 & Fuengirola (Málaga) \\
\hline 1997 & Barcelone \\
\hline 1998 & Almódovar del Río (Cordoue) \\
\hline 1999 & $\begin{array}{l}\text { Murcie } \\
\text { Benalmádena (Málaga) }\end{array}$ \\
\hline 2000 & Valence \\
\hline 2001 & $\begin{array}{l}\text { Jerez de la Frontera (Cadiz) } \\
\text { Sant Feliu de Guíxols (Gérone) }\end{array}$ \\
\hline 2002 & $\begin{array}{l}\text { La Puebla de Don Fadrique } \\
\text { Manresa (Barcelone) }\end{array}$ \\
\hline 2004 & Lucena (Cordoue) \\
\hline 2007 & $\begin{array}{l}\text { Riba-roja de Turia (Valence) } \\
\text { Las Palmas de Gran Canaria }\end{array}$ \\
\hline 2008 & $\begin{array}{l}\text { Bilbao-Derio } \\
\text { Palma de Majorque } \\
\text { Santa Cruz de Tenerife }\end{array}$ \\
\hline 2009 & Órgiva (Grenade) \\
\hline 2010 & Logroño \\
\hline 2011 & Calonge (Gérone) \\
\hline & parenthèses, la date de réouverture \\
\hline
\end{tabular}

10 Maria Rosa de Madariaga (2002) consacre un chapitre à la question des services (religieux, de nourriture, d'attention sanitaire, de divertissement) offerts aux troupes marocaines par l'armée de Franco. L'administration militaire a créé une série d' " hôpitaux musulmans " (ou "d'hôpitaux pour les Marocains ") qui ont souvent un petit cimetière adjacent. Madariaga cite des hôpitaux musulmans à Séville, Grenade, Jerez de la Frontera, Sanlucar de Barrameda, Cádiz, Villafranca de los Barros, Zafra, Plasencia, Toledo, Medina del Campo, Saragosse, Salamanque, Madrid, Leganés, Avilés et Griñon. 
La reconnaissance de ces lieux d'inhumation est le résultat de nombreuses demandes faites, localement, par des musulmans, afin de disposer d'un carré réservé au cimetière municipal. D'autres demandes sont en négociation avec des gouvernements locaux et I'on peut penser que dans un futur proche, le nombre de parcelles réservées pourrait augmenter.

Tableau 2 : Typologie des espaces d'inhumation musulmane en Espagne

\begin{tabular}{|c|c|c|c|c|}
\hline Typologie & Exemples & Histoire & Statut administratif & Situation \\
\hline I & Ceuta & $\begin{array}{l}\text { Le premier } \\
\text { cimetière connu. } \\
\text { Les musulmans de } \\
\text { cette ville y sont } \\
\text { enterrés }\end{array}$ & $\begin{array}{l}\text { Gestion municipale } \\
\text { avec les } \\
\text { musulmans locaux }\end{array}$ & $\begin{array}{l}\text { Cimetière } \\
\text { indépendant }\end{array}$ \\
\hline II & $\begin{array}{l}\text { Grenade } \\
\text { Séville } \\
\text { Saragosse } \\
\text { Burgos } \\
\text { León }\end{array}$ & $\begin{array}{l}\text { Issus de la Guerre } \\
\text { Civile et aujourd'hui } \\
\text { réouverts }\end{array}$ & $\begin{array}{l}\text { Gestion municipale } \\
\text { avec les } \\
\text { musulmans locaux }\end{array}$ & Carrés réservés \\
\hline III & $\begin{array}{l}\text { Melilla } \\
\text { Cordoue } \\
\text { Barcelone } \\
\text { Valence } \\
\text { Bilbao** }\end{array}$ & $\begin{array}{l}\text { Carrés réservés } \\
\text { dans des cimetières } \\
\text { municipaux }\end{array}$ & $\begin{array}{l}\text { Gestion municipale } \\
\text { avec les } \\
\text { musulmans locaux }\end{array}$ & $\begin{array}{l}\text { Carrés réservés, } \\
\text { avec une séparation } \\
\text { formelle à } \\
\text { I'exception de } \\
\text { Melilla où le } \\
\text { cimetière est } \\
\text { indépendant }\end{array}$ \\
\hline IV & $\begin{array}{l}\text { Griñon*** } \\
\text { Fuengirola } \\
\text { La Puebla de don } \\
\text { Fadrique }\end{array}$ & Cimetières privés & Gestion privée & $\begin{array}{l}\text { Cimetières } \\
\text { indépendants, } \\
\text { souvent adjoints à } \\
\text { une mosquée ou un } \\
\text { centre islamique }\end{array}$ \\
\hline
\end{tabular}

**Il convient de rajouter, Almódovar del Río, Murcie, Benalmádena, Jerez de la Frontera, Manresa, Lucena, Riba-roja de Turia, Las Palmas de Gran Canaria, Palma de Majorque, Órgiva, Logroño, SantFeliu de Guíxols *** Griñon fût cédé par l'armée espagnole au Consulat du Maroc à Madrid

\section{Cimetières historiques et récents}

Dans cette partie, nous allons rendre compte de huit expériences de création, d'aménagement et de gestion de parcelles réservées aux sépultures musulmanes dans quatre cimetières " historiques " et dans quatre cimetières récents.

\section{Le cimetière de Sidi Embarek à Ceuta}

Le cimetière de Sidi Embarek à Ceuta est le lieu d'inhumation musulmane le plus ancien d'Espagne. Datant du XIXe siècle, il porte le nom de la tombe du marabout (XVIIle siècle), près de laquelle les musulmans pieux ont été enterrés dans la tradition de l'Afrique du Nord. Les tombes les plus anciennes sont situées près de celle du marabout, les plus récentes sur des terrasses adjacentes. Jusqu'à la fin du XXe siècle, les pratiques funéraires islamiques sont restées privées, donnant lieu à des cérémonies familiales ${ }^{11}$.

Le cimetière de Sidi Embarek est une unité indépendante au sein du Département 
de Santé publique de la ville autonome, grâce à la signature d'un accord avec l'Association de la Mosquée Noor qui prend en charge l'organisation et la gestion du cimetière depuis les années 1980. Aujourd'hui, le vieux cimetière situé à la périphérie de la tombe du marabout a été fermé pour cause de saturation. Par contre la salle des funérailles, le magasin et la maison du moqaddem, en charge de l'entretien du cimetière, de l'assistance aux familles des défunts et des prières funèbres, sont toujours utilisés.

Le cimetière actuel utilise les terrasses qui sont situées à l'opposé des terrains de I'ancien cimetière. Le bureau se trouve dans le quartier voisin de La Reina, siège administratif qui gère et archive les données de toutes les tombes au Sidi Embarek. Toutefois, le cimetière actuel est insuffisant pour les besoins de la communauté musulmane de la ville (depuis 2000, on recense une moyenne d'environ vingt ou trente enterrements par mois). Il est prévu de l'agrandir par un nouveau domaine de $13000 \mathrm{~m}^{2}$ qui reliera la partie ancienne à celle utilisée actuellement.

Les tombes y sont conformes à la tradition nord-africaine selon laquelle le périmètre est marqué par de petits murs en pierre blanchis à la chaux. Si les plus anciennes ont perdu l'identification de la personne décédée, les plus récentes portent un nom et une petite légende en arabe, ou bien un numéro d'enregistrement. Sidi Embarek est un cimetière " vivant " et grâce à sa proximité avec des quartiers historiques musulmans, il reçoit tous les jours de nombreux habitants de la ville qui rendent visite au marabout, mais aussi aux tombes des parents et amis.

\section{Le cimetière de Séville}

La parcelle islamique se trouve dans le cimetière de San Fernando, ouvert en 1936, pendant la guerre civile espagnole, afin d'y déposer les corps des soldats marocains qui faisaient partie de l'armée nationale (Valencia, 1995 ; Tarrés, 2006). Cette parcelle se situe dans l'angle nord-ouest du cimetière, à côté du mur extérieur et elle a une entrée indépendante. Après la guerre civile, en 1944, la mairie de Séville a fermé le cimetière, en affirmant qu'il n'était plus utilisé. Cette zone est ensuite devenue un entrepôt pour l'entretien général du cimetière. En 1984, la Communauté islamique de Séville-Umma (une organisation composée principalement de musulmans convertis) a demandé au conseil municipal d'abroger cette fermeture. Après diverses initiatives, propositions et négociations, le Conseil a accepté en 1987 de rouvrir la parcelle qui a été dénommée " cimetière musulman " dont il a octroyé la gestion à perpétuité à la Communauté islamique de Séville-Umma.

Le carré musulman du cimetière San Fernando occupe environ $200 \mathrm{~m}^{2}$ avec une capacité de quarante-huit sépultures adultes. Dans le plan officiel du cimetière, cette section est appelée " cimetière arabe ". On trouve un espace pour le lavage rituel du corps du défunt et les tombes sont orientées perpendiculairement à l'entrée de la parcelle.

L'accord signé entre la mairie et la communauté islamique détermine que le conseil municipal sera déchargé de la responsabilité de la gestion administrative, tandis que la communauté devra entretenir la parcelle et contrôler l'accès, en demandant un certificat shahada (profession de foi musulmane). Seuls les musulmans vivant à Séville pourront être enterrés dans ce cimetière. Les funérailles auront lieu sur place et I'on n'utilisera pas de cercueil, conformément à la réglementation en vigueur dans la Communauté autonome d'Andalousie. Ce cimetière est maintenant proche de la saturation, mais la municipalité n'envisage pas de l'étendre. 


\section{Le cimetière de Grenade}

Le cimetière de Grenade a été fondé à l'époque de la guerre civile pour la même raison que celui de Séville : inhumer les soldats marocains défunts. En 1937, la ville de Grenade a demandé au gouvernement provisoire de Burgos l'autorisation d'enterrer les corps de vingt soldats marocains dans le "Llano de la Perdiz ", un terrain à côté du cimetière de San José qui fait partie de la Dehesa del Generalife, patrimoine historique de l'État, là où se situe l'Alhambra ${ }^{12}$. Après la guerre, le cimetière a cessé de fonctionner. Ce n'est qu'en 1972 qu'un imam d'Alicante a voulu être enterré dans le cimetière à côté de l'Alhambra. À partir de ce moment-là, plusieurs défunts y ont été enterrés, souvent sans que les autorités municipales le sachent. À partir de 1978, la mairie de Grenade a commencé à recevoir les premières demandes officielles des représentants musulmans de la ville pour que le cimetière soit rouvert. À la fin des années 1980, la mairie a accepté de fermer le cimetière par une clôture, mais sans résoudre son statut juridique.

Il a fallu attendre la fin des années 1990 pour que la mairie décide de remédier à la situation, en même temps qu'elle tentait de résoudre d'autres questions liées à la présence musulmane dans la ville, comme la construction de la mosquée dans le quartier d'Albayzin (Rosón, 2008). Un accord de collaboration, signé en 2002, a déterminé que la gestion administrative du cimetière était désormais partagée entre cimetière de San José et le Conseil islamique de Grenade. Ce dernier devait payer à la mairie le coût annuel de chaque tombe, mais pas celui de la cession du terrain dans le Llano de la Perdiz.

Fin 2009, la réforme du cimetière, nommé " Rauda de Grenade », est entrée en vigueur. Depuis son aménagement, celui-ci occupe une surface totale de $4970 \mathrm{~m}^{2}$ dont environ $676 \mathrm{~m}^{2}$ sont occupés par des tombes. D'une capacité de 570 tombes, en juillet 2010 , il en comptait 139 dont quatre-vingts identifiées. Parmi elles, douze sont celles de nouveau-nés ou de foetus. En plus de l'amélioration de l'entrée, le cimetière a été doté d'une chambre servant à l'ablution rituelle des corps. La clôture extérieure a également été renforcée. Les visites sont gérées par des responsables musulmans et désormais le carré musulman est mentionné sur le plan officiel du cimetière de San Jose. Depuis 2002, ceux qui souhaitent y être enterrés doivent contacter les responsables pour les formalités nécessaires et s'acquitter de 300 euros. Seuls peuvent y être enterrés les musulmans vivant dans la région de Grenade, ou ceux qui sont morts dans la ville. Cette mesure a pour but de limiter les demandes symboliques (comme celle du converti autrichien Muhammad Asad qui y fut inhumé en 1992).

\section{Le cimetière de Griñon (Madrid)}

Lieu d'inhumation des soldats marocains de l'armée du général Franco, le cimetière de Griñon à Madrid reste aujourd'hui le seul espace où enterrer les défunts musulmans du centre de l'Espagne. Ce carré, situé à côté du cimetière de la ville de Griñon (9 000 habitants, à vingt-sept kilomètres de Madrid), résulte d'un don privé à l'armée, destiné à enterrer les soldats musulmans pendant la guerre civile. Ce n'est que dans les années 1970 que le ministère de la Défense confia sa gestion au Consulat du Maroc à Madrid. Depuis, ce cimetière accueille les musulmans résidant à Madrid et dans les communautés de Castilla-La Mancha et de Castille-Leon, quelle que soit leur origine nationale.

Bien que le cimetière de Griñon occupe environ $7000 \mathrm{~m}^{2}$, il ne suffit pas pour une moyenne annuelle de 200 défunts. On estime qu'il contient actuellement 2800 tombes

12 Ensemble de palais datant de I'occupation musulmane du VIIIle au XVe siècle. 
alors qu'il est d'une capacité de 3600 . Les musulmans de la région ont critiqué publiquement la mauvaise gestion et le mauvais entretien du cimetière par le Consulat qui s'est vu contraint d'enlever des tombes anciennes afin de disposer de nouveaux emplacements. Ce cimetière dispose d'une petite salle de prières gérée par un imam rémunéré par le Consulat. Les tombes sont orientées vers la Mecque et les défunts sont placés dans un cercueil. Le Consulat interdit les images des défunts et veille à ce que les tombes restent modestes.

Le paradoxe de ce cimetière est que la plupart des inhumés, convertis espagnols, sont originaires de pays comme I'Irak, I'Iran, le Pakistan ou l'Égypte tandis que les Marocains préfèrent en majorité être rapatriés vers leur région d'origine.

\section{Le cimetière de Barcelone}

À Barcelone, la demande d'une parcelle réservée aux musulmans a une longue histoire. Elle est redevenue d'actualité après la signature de l'Accord de 1992 répondant aux demandes faites par les représentants musulmans (Moreras, 1999). En effet, le gouvernement de la ville de plus en plus conscient de l'émergence d'une hétérogénéité sociale et culturelle liée à l'installation d'immigrés suggère dans son Programme Interculturel (1996) d'adapter la réglementation relative aux cimetières aux nouvelles réalités des groupes non chrétiens présents dans la ville. Ce n'est qu'en 1997 que le conseil municipal signe un accord avec le Centre Islamique de Formation Religieuse, I'association Pakistanaise Wright Protection Association, I'association catalane-palestinienne et I'association catalane-libanaise qui laissent à ces quatre associations la prise en charge du coût de la construction des tombes (environ 24000 euros).

L'accord supposait la cession d'une parcelle de $552 \mathrm{~m}^{2}$ dans le cimetière de Collserola, mais excluait la création d'un carré spécifique (comme celui des juifs). II s'agissait seulement d'un petit périmètre, avec une simple séparation formée par une rangée de cyprès. L'accord précise également que les rituels d'ablution des corps doivent être réalisés avant le déplacement du cadavre au cimetière pour l'inhumation ${ }^{13}$.

Le Centre Islamique de Formation Religieuse prend en charge la gestion des tombes, ce qui a d'ailleurs provoqué une vive controverse chez les musulmans locaux qui ont remis en question les tarifs pratiqués par le Centre pour chaque décès ainsi que le mode d'inhumation qui rend obligatoire l'usage du cercueil et prévoit la possibilité de déplacer les restes humains.

II convient de signaler que pendant longtemps, cette parcelle a été l'unique lieu d'inhumation en accord avec les règles islamiques en Catalogne. En conséquence, plus de la moitié des personnes enterrées dans ce cimetière ne résidaient pas à Barcelone. Depuis 2011 la direction du cimetière de Collserola essaie de modifier les conditions de l'accord de 1997 avec les représentants musulmans à Barcelone, afin de modifier la gestion de cette parcelle.

13 En 2007, I'autre grand cimetière de Barcelone, Montjuic, a ouvert une salle spécifique pour l'ablution des défunts musulmans. L'inhumation du cadavre y est faite en utilisant un cercueil. 


\section{Le cimetière Suhail (Fuengirola, Málaga)}

Le cimetière privé de Fuengirola a été inauguré en 1996, à côté du cimetière municipal de la ville de Fuengirola. Sa gestion est assurée par la Communauté Islamique Suhail et par l'entreprise de pompes funèbres Empresa Funeraria. Ce cimetière offre un espace de plus de $5000 \mathrm{~m}^{2}$, avec tous les services funéraires et de sépulture pour les musulmans : procédures administratives, salles d'attente, zone pour les pratiques funéraires, mosquée de $300 \mathrm{~m}^{2}$, parking et aire d'inhumation. La nécropole est urbanisée et organisée en blocs distincts, combinant les jardins et les tombeaux, conformément à la loi andalouse. On trouve un espace réservé aux enterrements des enfants, qui sont les plus fréquents. Du point de vue esthétique, le cimetière Suhail est très austère : les tombes disposent d'une petite stèle funéraire portant le nom du défunt (dans certains cas, un simple numéro) et quelques inscriptions, la plupart écrites en langue arabe.

Le cimetière accueille un nombre élevé de défunts qui sont transférés d'autres régions d'Andalousie et du reste de l'Espagne, soit parce qu'ils ne peuvent pas être inhumés dans leur lieu de résidence soit parce qu'ils ne font pas confiance aux responsables des cimetières locaux.

\section{Le cimetière de Bilbao}

Le cimetière de Derio-Zamudio (il appartient à ces deux villes) est le principal cimetière municipal de Bilbao. D'une superficie de $208000 \mathrm{~m}^{2}$, il abrite presque 28600 tombes. La création d'une parcelle musulmane résulte de l'accord signé en octobre 2008 entre les services funéraires de Bilbao et I'Union des Communautés Islamiques du Pays Basque (UCIPV) qui souhaitaient que les musulmans disposent d'un espace pour inhumer leurs morts. Dans son rapport annuel de 2008, I'Ararteko (le médiateur de la république au Pays Basque espagnol) a élaboré une recommandation destinée aux cimetières municipaux afin qu'ils favorisent la création d'espaces réservés aux inhumations islamiques.

La parcelle de $450 \mathrm{~m}^{2}$, avec une capacité d'environ cinquante tombes, est située dans la partie nord du cimetière de la ville de Derio. L'espace est discret, accessible par une porte métallique fermée et sans aucune indication particulière. Cette discrétion a été voulue et précisée dans I'accord entre I'UCIPV et I'Union des communautés qui est la seule responsable de l'accès à cette parcelle. En outre le plan officiel du cimetière ne mentionne pas la caractéristique du lieu. Cette relative invisibilité relève aussi d'une décision prise par l'administration du cimetière " soucieuse d'assurer la sécurité " ${ }^{14}$.

L'accord signé avec I'UCIPV établit le droit d'utiliser ce carré pendant soixante-quinze ans. Pour chaque année de concession, la communauté musulmane doit s'acquitter de la somme de 5 607,48 euros. Le cimetière de Derio a dû assumer des travaux pour l'aménagement de la parcelle, financer son entretien et assurer la gestion administrative des inhumations ${ }^{15}$. Le cimetière municipal prend en charge la préparation et l'excavation des tombes, tandis que les membres de I'UCIPV sont responsables de la préparation et de la manipulation des cadavres, en conformité avec les règlements d'hygiène. Désormais et suite aux accords, seuls pourront être inhumés les musulmans nés, résidents ou décédés dans la ville de Bilbao.

\footnotetext{
14 Le cimetière municipal n'offre aucun équipement pour l'ablution rituelle du cadavre. Les tombes sont entourées par des bordures de petite taille pour éviter que les visiteurs ne marchent dessus. Jusqu'en décembre 2010 (date de notre enquête), huit fœtus et bébés ont été enterrés dans cette parcelle, mais aucun adulte.

15 La taxe à payer pour l'entretien annuel de la parcelle est de dix-neuf euros et quatre-vingt-deux centimes pour chaque tombe utilisée.
} 


\section{Le cimetière de Manresa}

Manresa est une ville moyenne (77 000 habitants), située au centre de la Catalogne, à cinquante-sept kilomètres de Barcelone. La demande de disposer d'une parcelle pour les inhumations musulmanes dans le cimetière municipal remonte à la création de la première mosquée dans la ville, au début des années 1990. Mais la demande formelle a été faite par la Communauté islamique de Bages en 1999. La négociation pour disposer d'une parcelle dans le cimetière municipal reposait sur le fait qu'un tel service ne devait concerner que les musulmans résidant dans la ville de Manresa. De plus, la zone réservée devait occuper une superficie de $136 \mathrm{~m}^{2}$ et pouvait accueillir vingt-neuf tombes. Les tombes devaient être construites en béton et mesurer très précisément deux mètres sur quatre-vingts centimètres.

La question la plus difficile à négocier a été l'accès à la parcelle. Les musulmans voulaient une entrée directe, sans avoir à traverser tout le cimetière catholique. La mairie s'opposait à cette demande, considérant que cela renforcerait le caractère exclusif de la parcelle, ce qui aurait été contraire au principe de sécularisation du cimetière. En définitive, il a été convenu d'utiliser une porte latérale d'accès, uniquement pour faire entrer les corps. Au départ, cette zone devait être séparée du reste du cimetière par des arbres, mais finalement un mur entourant la parcelle a été construit ${ }^{16}$.

\section{Conclusion : l'activation des ressources communautaires}

Les huit cas analysés ici détaillent un processus d'adéquation administrative des pratiques funéraires musulmanes en Espagne. Les recommandations élaborées par des entités publiques comme la Fondation Pluralisme et Coexistence du Ministère de la Justice ${ }^{17}$, favorable à la création de nouveaux carrés musulmans, s'explique par I'application d'un cadre légal qui garantit le droit à être enterré en accord avec les convictions philosophiques et religieuses de chacun. Les administrations locales adoptent ces recommandations selon l'espace disponible dans les cimetières municipaux et selon la formalisation des demandes de la part des représentants musulmans.

Ces recommandations jouent en faveur de la reconnaissance à la spécificité des rites funéraires islamiques et de l'incorporation de la diversité des pratiques et des cérémonies religieuses que développent thanatopracteurs et entreprises funéraires. Joue également un présupposé sous-jacent reliant l'inhumation en terre espagnole à l'émergence d'un islam espagnol. La création de ces carrés musulmans est comprise comme un élément fondamental du processus de " naturalisation " des musulmans en Espagne.

Cependant, la gestion communautaire de la mort chez les Marocains en Espagne ne se limite pas à l'usage des carrés réservés dans les cimetières municipaux, car la majorité des défunts sont rapatriés dans leurs régions d'origine. En conséquence, nombre de ces carrés sont partiellement occupés et peu saturés, excepté quelques cas particuliers. Actuellement, les demandes les plus nombreuses concernent les enterrements de fœtus et de nouveau-nés, des sépultures qui n'avaient pas été prévues dans ces carrés.

16 Le cimetière municipal a proposé deux modalités de concession à la Communauté islamique de Bages responsable de la gestion de ces tombes : de cinq et cinquante ans, avec des frais de 405 et 1160 euros respectivement. L'investissement initial, de 33700 euros, fait par le conseil municipal, devra être remboursé à la mairie par la Communauté islamique de Bages. Le cimetière municipal met également à la disposition des musulmans ses services en vue de préparer l'enterrement des corps. La signature définitive de l'accord a eu lieu en novembre 2002.

17 Voir le site web de la Fondation Pluralisme et Coexistence (www.pluralismoyconvivencia.es). 
Parler de "gestion communautaire de la mort " des migrants marocains renvoie à leur responsabilité collective face à la mort d'un des leurs. Définir une " bonne mort ", celle qui correspond aux normes prévues par la tradition (Thomas, 1975), consiste aussi à comprendre comment des collectifs régulent la vie des membres d'un groupe social, en supposant qu'il existe une continuité logique entre vie et mort. Or, vivre loin du pays d'origine, en situation de migration, génère des circonstances qui peuvent altérer cette continuité. Le décès d'un migrant peut provoquer une " mauvaise mort ", ou mort anomique, qui aura un effet de division et de perturbation du groupe social. Ainsi, la solidarité autour du cadavre se transforme en une preuve de la capacité du groupe à se réguler.

L'activation de ressources du groupe social pour financer les frais de transfert du cadavre vers le pays d'origine (spontanément au moment du décès, ou à partir de fonds mis en commun) est l'option préférée des migrants marocains (mais aussi sénégalais, pakistanais et mauritaniens) plutôt que les carrés des cimetières municipaux. Même quand ils sont bien installés en Espagne, les migrants continuent de contracter des assurances de rapatriement des corps. On pourrait y voir un effet du principe de reconstruction généalogique, une façon de " respecter l'ordre généalogique en enterrant dans les tombes de la famille d'origine "(Aggoun, 2006 : 77). De notre point de vue, le sentiment qu'exprime le choix du transfert vers le lieu de naissance est davantage un souci de filiation nationale et culturelle que religieuse. La mort devient la dernière occasion de se rattacher à I'histoire familiale, malgré la transgression du principe islamique qui veut que le croyant soit enterré là où il a trouvé la mort. Le corps est " avant tout un support généalogique, une preuve de l'identité " (Chaïb, $2000: 23$ ) et la mort une possibilité "d'arrangement généalogique " et de " réappropriation de son itinéraire biographique " (Chaïb, ibid.). Une volonté qui se nourrit souvent de la peur de mourir loin du cercle familial et d'être ensuite oublié et absent de la mémoire familiale.

En définitive, il convient d'analyser le rôle joué par les carrés musulmans au regard du rapatriement des corps, option favorite de la plupart des migrants musulmans en Espagne, qui mobilisent leurs ressources sociales dans ce sens. Mais il faut aussi y voir une expression de la manière dont la société espagnole considère la pluralité des formes funéraires qui s'imposent progressivement dans les cimetières municipaux.

\section{Références bibliographiques}

Aggoun Atmane (2006) Les musulmans face à la mort en France, Paris, Espace ÉthiqueÉditions Vuibert, 156 p.

Baudry Patrick (1999) La place des morts. Enjeux et rites, Paris, L'Harmattan, 206 p.

Chaïb Yassine (2000) L'émigré et la mort, Marseille, CIDIM / Edisud, 256 p.

Jiménez Lozano José (1978) Los cementerios civiles y la heterodoxia española, Madrid, Taurus, $376 \mathrm{p}$.

Madariaga Rosa Maria de (2002) Los moros que trajo Franco. La intervención de tropas coloniales en la guerra civil, Barcelona, Ediciones Martínez Roca, 442 p.

Moreras Jordi (2011) Spain, in Jorgen S. Nielsen, Samim Akgönül, Ahmet Alibasic, Hugh Goddard and Brigitte Maréchal Eds., Yearbook of Muslims in Europe (vol. 2), Leiden, E. J. Brill, pp. 529-544. 
Moreras Jordi (2004) Morir lejos de casa: la muerte en contexto migratorio, in Bernabé López García y Mohamed Berriane Eds., Atlas de la inmigración marroquí en España, Madrid, Universidad Autónoma de Madrid-Taller de Estudios Internacionales Mediterráneos, pp. 427-429.

Moreras Jordi (1999) Musulmanes en Barcelona. Espacios y dinámicas comunitarias, Barcelona, CIDOB Edicions, 397 p.

Motilla Agustín (2004) La protección de los lugares de culto islámicos, in Agustín Motilla Ed., Los musulmanes en España. Libertad religiosa e identidad cultural, Madrid, Trotta, pp. 79-106.

Rodriguez Blanco Miguel (2010) Libertad religiosa y cementerios (primeras aproximaciones), Anuario de Derecho Eclesiástico del Estado, 26, pp. 591-608.

Rosón Javier (2008) ¿El retorno de Tariq? Comunidades etnoreligiosas en el Albayzín granadino, Thèse de doctorat, Dept. Anthropologie Sociale, Université de Grenade.

Sayad Abdelmalek (2000) Préface, in Yassine Chaïb, L'émigré et la mort, Marseille, CIDIM / Edisud, pp. 5-16.

Tarrés Sol (2010) Musulmanes en Andalucía, in Rafael Briones Ed., ¿Y tú (de) quién eres? Minorías religiosas en Andalucía, Madrid, Fundación Pluralismo y Convivencia-Icaria Editorial, pp. 289-347.

Tarrés Sol (2006) Ritos funerarios en el islam: la praxis entre los musulmanes de Sevilla, Zainak, 28, pp. 429-446.

Tarrés Sol, Solé Ariadna, Jàvega Neus y Moreras Jordi (2012) Migrar, morir, ¿retornar? Un programa de investigación sobre la muerte en contexto migratorio, VII Congreso sobre las Migraciones Internacionales en España (Bilbao, avril 2012).

Thomas Louis-Vincent (1975) Anthropologie de la mort, Paris, Payot, 538 p.

Valencia Rafael (1995) El cementerio musulmán de Sevilla, 'llu. Revista de Ciencias de las Religiones, 0, pp. 263-270.

Viguera Maria Jesús (1995) Al-Andalus como interferencia, in Montserrat Abumalham Ed., Comunidades islámicas en Europa, Madrid, Trotta, pp. 45-62. 


\section{Jordi Moreras et Sol Tarrés}

\section{Les cimetières musulmans en Espagne : des lieux de l'altérité}

Tout au long de l'histoire, les cimetières ont été constitués comme des lieux pluriels du point de vue culturel et religieux. L'histoire agitée des cimetières en Espagne reflète l'évolution socio-politique du pays et peut être comprise comme une série de réponses données à " l'autre mort " (celle qui diffère du canon catholique) la plaçant dans ou hors les murs du cimetière. Récemment, la diversité religieuse croissante de la société espagnole commence également à se manifester dans les espaces d'inhumation, exigeant de nouvelles alternatives de gestion dans le traitement de la mort. Dans le cas des cimetières musulmans, l'administration de la diversité funéraire est conditionnée par le fait qu'une partie importante de ces cimetières a été créée au cours de la guerre civile espagnole afin d'inhumer les corps des soldats marocains qui ont combattu dans l'armée du général Franco. Cet article compare les différents espaces funéraires musulmans en Espagne et analyse les pratiques contemporaines de leur gestion.

\section{The Muslim Cemeteries in Spain: Spaces of Otherness}

Throughout history, cemeteries have been established as culturally and religiously diverse places. The history of cemeteries in Spain, reflecting the changing socio-political circumstances, can be understood as a series of responses in relation to diverse death (which differs from the Catholic canon), placing it intra or extramural of the cemetery grounds. The increasing religious diversity of Spanish society also begins to be evident in burial spaces, demanding new management alternatives in the treatment of death. In the case of Muslim cemeteries, the administrative management of diversity of funeral practices, is mixed with historical precedent since a significant portion of these cemeteries were created during the Spanish Civil War to bury the Moroccan soldiers who fought in the army of general Franco. This paper compares some different Islamic burial spaces in Spain, analysing its contemporary practices of management.

\section{Los cementerios musulmanes en España: otros espacios de la alteridad}

A lo largo de la historia, los cementerios se han constituido como espacios cultural y religiosamente diversos. La agitada historia de los cementerios en España, reflejo de las circunstancias socio-políticas cambiantes, puede ser entendida como una sucesión de respuestas en relación a la muerte diversa (la que difiere del canon católico), emplazando ésta intra o extramuros del recinto del cementerio. La reciente diversidad religiosa de la sociedad española también comienza a hacerse patente en los espacios funerarios, reclamando nuevas alternativas de gestión en el tratamiento de la muerte. En el caso concreto de los cementerios musulmanes, esta gestión administrativa de la diversidad funeraria, se entremezcla con el precedente histórico de que una significativa parte de estos cementerios fueron creados durante la guerra civil española para enterrar a los soldados marroquíes que combatieron en el ejército de Franco. Este texto compara diferentes espacios funerarios musulmanes en España, analizando las prácticas contemporáneas de su gestión. 